\title{
EDITORIAL
}

\section{Sniff nasal inspiratory pressure: simple or too simple?}

\author{
J-W. Fitting
}

$\mathbf{R}$ espiratory muscle weakness, be it of acute or chronic onset, is a potentially threatening condition. Weakness of inspiratory muscles generates an imbalance between muscle load and capacity that, when severe enough, leads to hypercapnic respiratory failure. Conversely, weakness of expiratory muscles impairs cough and airway clearance and favours lung atelectasis and infection. Dysfunction of both respiratory muscle groups commonly precipitates acute respiratory failure in neuromuscular disorders. In recent years, the importance of respiratory muscle assessment has been recognised and a variety of tests has been proposed $[1,2]$.

The strength of inspiratory muscles can be assessed either by volitional or by nonvolitional tests. The volitional tests are simple, portable and inexpensive. Their main limitation lies in their dependence on maximal voluntary neuromuscular activation, which, in practice, is difficult to ascertain. In contrast, the cortical motor command is bypassed by nonvolitional tests such as phrenic nerve magnetic stimulation [3]. Phrenic nerve stimulation offers the most reliable measure of diaphragm contractility, but is not widely available because it requires expensive equipment. It must be added that phrenic nerve stimulation may overestimate the diaphragm strength that is actually available to the patient in case of upper motor neuron lesions [4]. Thus, notwithstanding their limitations, volitional tests remain on the first line and must be best exploited.

Maximum inspiratory pressure $(P \mathrm{I}, \max )$ is the classic volitional test of inspiratory muscle strength. It is measured as the highest mouth pressure sustained for $1 \mathrm{~s}$ during a maximum inspiratory effort against a quasi occlusion. Although simple in principle, the PI,max manoeuvre is difficult for many and requires a hermetic seal around the mouthpiece. As a consequence, low values may be due to true muscle weakness, a submaximal effort, or air leaks in the case of facial muscle weakness. The sniff is an alternative manoeuvre that is more natural and easier for most subjects. During a maximal sniff, there is strong activation of the diaphragm and of the scalene muscles [5, 6]. Thus, the sniff has proved valuable to assess diaphragm strength using transdiaphragmatic pressure (sniff $P$ di), or global inspiratory muscle strength using oesophageal pressure (sniff Poes) [7,8]. More recently, the method of sniff nasal inspiratory pressure (SNIP) was proposed as a noninvasive test of inspiratory muscle strength [9]. This very simple procedure consists of measuring peak nasal pressure in one occluded nostril during a maximal sniff performed from

CORRESPONDENCE: J-W. Fitting, Service de Pneumologie, Centre Hospitalier Universitaire Vaudois, 1011 Lausanne, Switzerland. Fax: 41 213141384. E-mail: jean-william.fitting@chuv.ch relaxed end-expiration through the contralateral patent nostril. During a vigorous sniff, the nasal valve of the patent nostril collapses and the pressure measured beyond the collapsed segment closely reflects oesophageal pressure and, therefore, inspiratory muscle strength. Various types of nasal plugs have been used in the past, but portable manufactured systems are now available for measuring SNIP. Severe nasal congestion represents a limitation of the method because it hinders pressure transmission and leads to falsely low values.

Reference values have been established for SNIP in adults [10] and children [11, 12]. Interestingly, SNIP is similar in children and adults, despite a large difference in respiratory muscle mass. This peculiarity is probably due to the predominant activation of the diaphragm, which acts as a piston in the thoracoabdominal cavity. The ratio of diaphragm muscle mass to the axially projected area of the diaphragm varies little from childhood to adulthood, and explains the relative stability of maximal Pdi across ages $[13,14]$. SNIP is often higher than $P$ I,max in healthy subjects. Initially, this is surprising, as a loss of force would be expected during such a dynamic manoeuvre due to the force-velocity relationship of muscles. This apparent paradox is probably explained by a more complete neuromuscular activation during the SNIP test, which is much easier to perform. However, the limits of agreement between these two tests are wide. This indicates that SNIP and PI,max are not interchangeable and should be considered as complementing one another for the assessment of inspiratory muscle strength.

In this issue of the European Respiratory Journal, LOFASO et al. [15] report a study assessing the number of sniffs that are necessary to obtain a true maximal SNIP. This is particularly relevant because the main limitation of volitional tests remains the uncertainty about the achieved neuromuscular activation. Earlier observations have suggested that the learning effect is completed within the first 10 maximal sniffs [10]. LOFASO et al. [15] asked a group of healthy subjects to perform 40 consecutive maximal sniffs and analysed them in sets of 10 . It was found that the best SNIP was 6\% higher in sniffs 11-20 than in sniffs $1-10$. The SNIP then reached a plateau after the twentieth sniff. The study was extended to a large group of patients performing 20 consecutive sniffs and, again, the best SNIP was $10 \%$ higher in the second set of 10 sniffs than in the first set. These results are remarkably consistent with some previous observations made in different settings. In a small group of patients with amyotrophic lateral sclerosis (ALS) who were examined repeatedly, the best of 20 SNIP values exceeded the best of the first 10 by $7 \%$ [16]. In a larger group of patients with asthma or various medical conditions, the best of 15 SNIP values was higher than the best of the first 10 by 
$10 \%$ [17]. Thus, it appears clear that the maximal SNIP will be achieved with greater certainty after 20 sniffs than after only 10. However, the gain is relatively small after the tenth trial and it may be questioned whether it is worth imposing this on the patient. To solve this dilemma, the authors proposed to perform $>10$ sniffs only when SNIP is slightly below normal or when it is used to monitor functional decline over time. This recommendation appears to be a reasonable compromise between scientific rigour and practical considerations. Some studies with a similar goal have recommended between nine and 20 trials for achieving a true $P_{I, \max }[18,19]$, but such long procedures have neither been adopted in clinical practice nor been endorsed by international guidelines [2].

What has been learned since the introduction of SNIP in to the clinical arena? This test appears particularly suited to neuromuscular weakness because it obviates the use of a mouthpiece and because it is easily mastered by the vast majority of patients. Among 126 young patients with neuromuscular or skeletal disorders, all could perform the SNIP, whereas 10 could not perform the PI,max [20]. Among 258 adult patients with neuromuscular weakness, eight were unable to perform the SNIP and nine the PI,max [21]. In patients with neuromuscular disorders, SNIP was found to be the main determinant of vital capacity [20]. However, patients with severe neuromuscular disorders may have difficulty in performing a rapid sniff, leading to a potential overestimation of muscle weakness by SNIP [21]. This limitation implies that the assessment of severe muscle weakness should not rely on SNIP only, but should include other tests like PI,max, vital capacity, nocturnal oximetry or arterial blood gases. In patients with restrictive thoracic disease treated with noninvasive ventilation, a correlation was found between the fall in the Epworth sleepiness score and the gain in sniff $P$ oes and SNIP, whereas no correlation existed with twitch $P$ di obtained by phrenic nerve stimulation [22]. This discrepancy may suggest that activation of respiratory muscles by volitional tests such as SNIP is hindered by excessive somnolence. Similarly, a reduced central command has been hypothesised to explain the fall in SNIP and PI,max documented during experimental hypobaric hypoxia [23].

The SNIP test has been used by different groups to assess patients with ALS. SNIP seems to be more frequently feasible than $P \mathrm{I}$,max in advanced disease $[16,24]$, but is often difficult to perform for patients with bulbar involvement [25]. SNIP appears to be of some value in monitoring the evolution of disease, showing a linear decline [16] and proving to be better than $P \mathrm{I}$,max and vital capacity in predicting hypercapnia $[4,25]$. As with other indices of respiratory muscle strength, SNIP showed moderate-to-strong correlations with different scores of quality of life in ALS [26]. Finally, a SNIP value $<40 \mathrm{cmH}_{2} \mathrm{O}$ was associated with a median survival of 6 months, and a value $<30 \mathrm{cmH}_{2} \mathrm{O}$ with a median survival of 3 months [24].

The transmission of rapid pressure changes from the alveoli to the upper airways is altered in the case of airflow limitation [27], and indeed, SNIP underestimated sniff $P_{\text {oes }}$ on average by $14 \%$ in patients with acute asthma [17] and by $19 \%$ in patients with stable COPD [28]. This limitation has to be considered and may explain part of the increase seen in SNIP after lung volume reduction surgery (LVRS) [29]. Nevertheless, after an initial gain, SNIP was shown to increase further 9 months after LVRS, when no further change occurred in forced expiratory volume in one second or functional residual capacity, suggesting a possible delayed muscle adaptation [30].

A knowledgeable author once advised: "Make everything as simple as possible, but not simpler." Simplicity is the main asset of sniff nasal inspiratory pressure, but it carries limitations. Due to its ease of use, sniff nasal inspiratory pressure proves valuable as a first-line tool for diagnosing respiratory muscle weakness. The well-founded and practical recommendations of LOFASO et al. [15] will help to disseminate this procedure more widely. Nevertheless, in some patients, inconclusive results with simple tests will require a formal assessment, including nonvolitional tests to confirm or refute respiratory muscle weakness.

\section{REFERENCES}

1 Polkey MI, Green M, Moxham J. Measurement of respiratory muscle strength. Thorax 1995; 50: 1131-1135.

2 American Thoracic Society, European Respiratory Society. ATS/ERS Statement on respiratory muscle testing. Am J Respir Crit Care Med 2002; 166: 518-624.

3 Similowski T, Fleury B, Launois S, Cathala HP, Bouche P, Derenne JP. Cervical magnetic stimulation: a new painless method for bilateral phrenic nerve stimulation in conscious humans. J Appl Physiol 1989; 67: 1311-1318.

4 Lyall RA, Donaldson N, Polkey MI, Leigh PN, Moxham J. Respiratory muscle strength and ventilatory failure in amyotrophic lateral sclerosis. Brain 2001; 124: 2000-2013.

5 Nava S, Ambrosino N, Crotti P, Fracchia C, Rampulla C. Recruitment of some respiratory muscles during three maximal inspiratory manoeuvres. Thorax 1993; 48: 702-707.

6 Katagiri M, Abe T, Yokoba M, Dobashi Y, Tomita T, Easton PA. Neck and abdominal muscle activity during a sniff. Respir Med 2003; 97: 1027-1035.

7 Miller JM, Moxham J, Green M. The maximal sniff in the assessment of diaphragm function in man. Clin Sci (Lond) 1985; 69: 91-96.

8 Laroche CM, Mier AK, Moxham J, Green M. The value of sniff esophageal pressures in the assessment of global inspiratory muscle strength. Am Rev Respir Dis 1988; 138: 598-603.

9 Heritier F, Rahm F, Pasche P, Fitting JW. Sniff nasal inspiratory pressure. A noninvasive assessment of inspiratory muscle strength. Am J Respir Crit Care Med 1994; 150: 1678-1683.

10 Uldry C, Fitting JW. Maximal values of sniff nasal inspiratory pressure in healthy subjects. Thorax 1995; 50: 371-375.

11 Rafferty GF, Leech S, Knight L, Moxham J, Greenough A. Sniff nasal inspiratory pressure in children. Pediatr Pulmonol 2000; 29: 468-475.

12 Stefanutti D, Fitting JW. Sniff nasal inspiratory pressure. Reference values in Caucasian children. Am J Respir Crit Care Med 1999; 159: 107-111.

13 McCool FD, Conomos P, Benditt JO, Cohn D, Sherman CB, Hoppin FG Jr. Maximal inspiratory pressures and dimensions of the diaphragm. Am J Respir Crit Care Med 1997; 155: 1329-1334. 
14 McCool FD, Benditt JO, Conomos P, Anderson L, Sherman CB, Hoppin FG Jr. Variability of diaphragm structure among healthy individuals. Am J Respir Crit Care Med 1997; 155: 1323-1328.

15 Lofaso F, Nicot F, Lejaille M, et al. Sniff nasal inspiratory pressure: what is the optimal number of sniffs? Eur Respir J 2006; 27: 980-982.

16 Fitting JW, Paillex R, Hirt L, Aebischer P, Schluep M. Sniff nasal pressure: a sensitive respiratory test to assess progression of amyotrophic lateral sclerosis. Ann Neurol 1999; 46: 887-893.

17 Stell IM, Polkey MI, Rees PJ, Green M, Moxham J. Inspiratory muscle strength in acute asthma. Chest 2001; 120: 757-764.

18 Fiz JA, Montserrat JM, Picado C, Plaza V, Agusti-Vidal A. How many manoeuvres should be done to measure maximal inspiratory mouth pressure in patients with chronic airflow obstruction? Thorax 1989; 44: 419-421.

19 Wen AS, Woo MS, Keens TG. How many maneuvers are required to measure maximal inspiratory pressure accurately. Chest 1997; 111: 802-807.

20 Stefanutti D, Benoist MR, Scheinmann P, Chaussain M, Fitting JW. Usefulness of sniff nasal pressure in patients with neuromuscular or skeletal disorders. Am J Respir Crit Care Med 2000; 162: 1507-1511.

21 Hart N, Polkey MI, Sharshar T, et al. Limitations of sniff nasal pressure in patients with severe neuromuscular weakness. J Neurol Neurosurg Psychiatry 2003; 74: 1685-1687.

22 Nickol AH, Hart N, Hopkinson NS, Moxham J, Simonds A, Polkey MI. Mechanisms of improvement of respiratory failure in patients with restrictive thoracic disease treated with non-invasive ventilation. Thorax 2005; 60 754-760.

23 Deboeck G, Moraine JJ, Naeije R. Respiratory muscle strength may explain hypoxia-induced decrease in vital capacity. Med Sci Sports Exerc 2005; 37: 754-758.

24 Morgan RK, McNally S, Alexander M, Conroy R, Hardiman O, Costello RW. Use of sniff nasal-inspiratory force to predict survival in amyotrophic lateral sclerosis. Am J Respir Crit Care Med 2005; 171: 269-274.

25 Chaudri MB, Liu C, Watson L, Jefferson D, Kinnear WJ. Sniff nasal inspiratory pressure as a marker of respiratory function in motor neuron disease. Eur Respir J 2000; 15: 539-542.

26 Bourke SC, Shaw PJ, Gibson GJ. Respiratory function vs sleep-disordered breathing as predictors of QOL in ALS. Neurology 2001; 57: 2040-2044.

27 Murciano D, Aubier M, Bussi S, Derenne JP, Pariente R, Milic-Emili J. Comparison of esophageal, tracheal, and mouth occlusion pressure in patients with chronic obstructive pulmonary disease during acute respiratory failure. Am Rev Respir Dis 1982; 126: 837-841.

28 Uldry C, Janssens JP, de Muralt B, Fitting JW. Sniff nasal inspiratory pressure in patients with chronic obstructive pulmonary disease. Eur Respir J 1997; 10: 1292-1296.

29 Teschler H, Stamatis G, el-Raouf Farhat AA, Meyer FJ, Costabel U, Konietzko N. Effect of surgical lung volume reduction on respiratory muscle function in pulmonary emphysema. Eur Respir J 1996; 9: 1779-1784.

30 Degano B, Brouchet L, Rami J, et al. Improvement after lung volume reduction surgery: a role for inspiratory muscle adaptation. Respir Physiol Neurobiol 2004; 139: 293-301. 